\title{
THE RADICALS OF A SEMIGROUP
}

BY

\section{REBECCA SLOVER}

ABSTRACT. This paper investigates various radicals and radical congruences of a semigroup. A strongly prime ideal is defined. It is shown that the nil radical of a semigroup is the intersection of all strongly prime ideals of the semigroup. Furthermore, a semigroup with zero element is nil if and only if it has no strongly prime ideals. We investigate the question of when the left and right radical congruence relations of various radicals are equal. Some theorems analogous to theorems concerning the radicals of rings are also proved.

1. Introduction. Just as in the case for rings, various radicals of semigroups have been studied. In this paper we shall examine further some properties of the nil radical of a semigroup.

Let $N(S)$ denote the nil radical of a semigroup $S$. An ideal $P$ of the semigroup $S$ is strongly prime if there exists a subsemigroup $M$ of $S$ such that $P$ is the largest ideal of $S$ not meeting $M$, i.e.,

$$
P=\left\{a \in S: S^{1} a S^{1} \cap M=\varnothing\right\} .
$$

A strongly prime ideal is prime. It is shown that $N(S)$ is the intersection of all strongly prime ideals of $S$. Furthermore, a semigroup $S$ with zero element is nil if and only if $S$ has no strongly prime ideals.

H. J. Hoehnke has used congruence relations effectively to study the structure of semigroups. We shall investigate the question of when the left and right radical congruence relations introduced by Hoehnke are equal. Hoehnke defines $\operatorname{rad}_{R} S$, the right nil radical congruence of a semigroup $S$. One can similarly define $\operatorname{rad}_{L} S$, the left nil radical congruence of a semigroup $S$. It is not true that $\operatorname{rad}_{L} S=\operatorname{rad}_{R} S$. In fact, if $S$ is a left zero semigroup then $\operatorname{rad}_{R} S$ is the universal relation while $\operatorname{rad}_{L} S$ is the identity relation.

The right nil radical congruence of a regular Rees matrix semigroup over a group with zero will be considered in detail. It will be shown that this congruence relation is completely determined by the subgroups of the group and the positions of the nonzero entries of the sandwich matrix. Furthermore, the right nil radical

Received by the editors October 5, 1973.

AMS (MOS) subject classifications (1970). Primary 20M10; Secondary 20M30, 16A21. Key words and phrases. Radicals of a semigroup, radical congruence, strongly prime ideal.

Copyright $\odot$ 1975, American Mathematical Society 
congruence of a regular Rees matrix semigroup equals the left nil radical congruence if and only if they are both the identity relation.

In the final section of this paper the right and left Jacobson radical congruences of $S$, denoted by $\overline{\operatorname{rad}}_{R} S$ and $\overline{\operatorname{rad}}_{L} S$ respectively, are defined. This congruence relation has been studied extensively (see [1], [4], [5], [8], [10], and [11]). It has been known that if $T$ is an ideal of a ring, then the Jacobson radical of $T$ equals the intersection of $T$ with the Jacobson radical of the ring. It is natural to expect a similar result for the congruence relation $\overline{\mathrm{rad}}_{R} S$. Indeed, it will be shown that if $T$ is an ideal of the semigroup $S$ then $\overline{\operatorname{rad}}_{R} T$ is the relation $\overline{\operatorname{rad}}_{R} S$ restricted to $T$. It will also be shown that if $T$ is an ideal of $S$ such that $\overline{\operatorname{rad}}_{R} S / T=\overline{\operatorname{rad}}_{L} S / T$ and $\overline{\operatorname{rad}}_{R} T=\overline{\operatorname{rad}}_{L} T$ then $\overline{\operatorname{rad}}_{R} S=$ $\overline{\operatorname{rad}}_{L} S$.

Finally, it is proved that if $S$ is a regular Rees matrix semigroup over a group with zero then $\overline{\operatorname{rad}}_{R} S$ is completely determined by the positions of the nonzero entries of the sandwich matrix and $\overline{\operatorname{rad}}_{R} S=\overline{\operatorname{rad}}_{L} S$. Thus, for such a semigroup, it is not necessarily true that the right nil radical congruence equals the right Jacobson radical congruence.

The author would like to express her appreciation to the referee, whose suggestions have substantially improved the paper.

2. The nil radical. Throughout this paper $S$ will always denote a semigroup and $S^{1}$ will be the semigroup obtained from $S$ by adjoining an identity element 1. An element $a \in S$ such that as $=a$ for each $s \in S$ is a left zero of $S$. The set $0_{L}(S)$ of all left zeros of $S$ is either void or is an ideal of $S$ contained in every ideal of $S$. If $0_{L}(S)$ is nonvoid then $S / 0_{L}(S)$, the factor semigroup of $S$ modulo $0_{L}(S)$, contains zero. Let nil $\operatorname{rad}\left(S / 0_{L}(S)\right)$ be the sum of all nil ideals of $S / 0_{L}(S)$. Let $N_{L}(S)$ be the ideal of $S$ such that $N_{L}(S) / 0_{L}(S)=$ nil $\operatorname{rad}\left(S / 0_{L}(S)\right)$. If $0_{L}(S)=\varnothing$, let $N_{L}(S)=\varnothing$. Note that nil $\operatorname{rad}\left(S / 0_{L}(S)\right)$ contains each nil left ideal and each nil right ideal of $S / 0_{L}(S)$.

One can similarly define $N_{R}(S)$, the right nil radical of $S$. It is well known that any left zero of $S$ must equal any right zero of $S$. Thus, if both $N_{L}(S)$ and $N_{R}(S)$ are nonvoid then they are equal. When this is the case, then the nil radical of $S$ will be denoted by $N(S)$.

LEMMA 2.1. A strongly prime ideal is prime.

Proof. Let $P$ be a strongly prime ideal of $S$ and let $M$ be a subsemigroup of $S$ such that $P$ is the largest ideal of $S$ not meeting $M$. Let each of $A$ and $B$ be an ideal of $S$ such that $A B \subseteq P, A \nsubseteq \subseteq P$, and $B \nsubseteq \subseteq$. Since $A \cup P$ and $B \cup P$ are ideals of $S$ properly containing $P$ then $(A \cup P) \cap M \neq \varnothing$ 
and $(B \cup P) \cap M \neq \varnothing$. Let $a \in(A \cup P) \cap M$ and $b \in(B \cup P) \cap M$. Since $P \cap M=\varnothing$ then $a \in A \cap M$ and similarly $b \in B \cap M$. Thus $a b \in A B \cap$ $M \subseteq P \cap M=\varnothing$, which is a contradiction. Therefore, if $A B \subseteq P$ then either $A \subseteq P$ or $B \subseteq P$.

DEFINITION. Let $P(S)$ be the set of all strongly prime ideals of a semigroup $S$. If $P$ is a strongly prime ideal of $S$ and $M$ is a subsemigroup of $S$ such that $P$ is the largest ideal of $S$ not meeting $M$ then we shall say that $M$ is a subsemigroup associated with $P$.

THEOREM 2.2. A semigroup $S$ with zero element is nil if and only if $P(S)$ is void.

Proof. Suppose that $S$ is nil. Let $P$ be a strongly prime ideal and let $M$ be a subsemigroup associated with $P$. Let $x$ be an element of $M$. Since $S$ is nil, there exists a positive integer $n$ such that $x^{n}=0$. Thus $x^{n} \in\{0\} \cap$ $M \subseteq P \cap M=\varnothing$, which is a contradiction. Thus if $S$ is nil then $P(S)$ is void.

Conversely, suppose that $S$ is not nil. Let $x$ be an element of $S$ which is not nilpotent. Let $X=\left\{x^{n}: n\right.$ a positive integer $\}$. Thus $X$ is a subsemigroup of $S$. Let $U=\{U: U$ is an ideal of $S$ such that $U \cap X=\varnothing\}$. Since $\{0\} \in U$, then $U$ is nonempty. Let $P=\bigcup_{U \in U} U$. Then $P$ is a maximal ideal of $S$ such that $P \cap X=\varnothing$. Thus $P$ is an element of $P(S)$.

THEOREM 2.3. If $S$ is a semigroup with zero element then $N(S)=$ $\bigcap_{P \in P(S)} P$.

Proof. Let $P$ be a strongly prime ideal with associated subsemigroup $M$. Suppose that $x \in[N(S) \cup P] \cap M$. Since $P \cap M=\varnothing$ then $x \in N(S) \cap M$. Since $N(S)$ is nil, there exists a positive integer $n$ such that $x^{n}=0$. Thus $x^{n} \in\{0\} \cap M \subseteq P \cap M=\varnothing$, which is a contradiction. Therefore $[N(S) \cup$ $P] \cap M=\varnothing$. Since $P$ is a maximal ideal of $S$ such that $P \cap M=\varnothing$ and since $P \subseteq N(S) \cup P$ then $N(S) \subseteq P$. Therefore $N(S) \subseteq \bigcap_{P \in P(S)} P$.

Conversely suppose that $a$ is an element of $S$ such that $a \notin N(S)$. Thus there exists an element $b \in S^{1}$ such that $(a b)^{n}$ is not zero for each positive integer $n$. Let $M=\left\{(a b)^{n}: n\right.$ a positive integer $\}$. Thus $M$ is a subsemigroup of $S$ such that $\{0\} \cap M=\varnothing$. Let $P$ be a maximal ideal of $S$ such that $P \cap M=\varnothing$. Thus $P$ is an element of $P(S)$ and $a \notin P$.

3. A comparison between the radicals of a ring and the nil radical of a semigroup. If $S$ is a ring, the relationships of the radicals of the ring $(S,+, \cdot)$ and the radical of the multiplicative semigroup $(S, \cdot)$ will now be studied.

DEfinition. If $(S,+, \cdot)$ is a ring, let $J(S)$ be the Jacobson radical of 
the ring $S$ and let $N(S)$ be the nil radical of the ring $S$. Let $M(S)$ be the sum of the nil right ideals of $S$. Thus $M(S)$ is a two-sided ideal of $S$ but it is not known whether $M(S)$ is nil.

As before, the nil radical of the multiplicative semigroup $(S, \cdot)$ will be denoted by $N(S)$.

THEOREM 3.1. If $S$ is a ring then $N(S) \subseteq N(S) \subseteq M(S) \subseteq J(S)$.

Proof. Since a nil ideal of the ring $S$ is a nil semigroup ideal, then $N(S) \subseteq N(S)$. To see that $N(S) \subseteq M(S)$ let $i$ be an element of $N(S)$. Thus $i S$ is nil. Let ( $i$ ) be the principal right ideal of the ring $S$ generated by $i$. Let $x \in(i)$. Thus there exists an integer $n$ and an element $s$ in $S$ such that $x=n i+i$ s. Since $x^{2}=i\left(n^{2} i+n s i+n i s+s i s\right) \in i S$ and since $i S$ is nil, then $x^{2}$ is nilpotent. Therefore $x$ is also nilpotent and $i \in(i) \subseteq M(S)$. Thus $N(S)$ $\subseteq M(S)$.

To see that $M(S) \subseteq J(S)$, let $m$ be an element of $M(S)$. Thus there exist a finite number of nil right ideals of $S, I_{1}, I_{2}, \cdots, I_{q}$, such that $m \in$ $\Sigma_{j=1}^{q} I_{j}$. Each nil right ideal of $S$ is contained in $J(S)$ (see [6, pp. 8 and 9]). Thus $m \in J(S)$ and, consequently, $M(S) \subseteq J(S)$.

An example of a ring $S$ such that $J(S) \neq N(S)$ will now be considered.

Let $\Phi\langle x\rangle$ be the ring of formal power series in one indeterminant with coefficients in a field. By [6, p. 21], J( $\Phi\langle x\rangle)$ is the ideal $(x)$. Since $\Phi\langle x\rangle$ is a ring with no nonzero divisors of zero, then $N(\Phi\langle x\rangle)=\{0\}$. Thus $J(\Phi\langle x\rangle) \neq$ $N(\Phi\langle x\rangle)$.

\section{The nil radical congruence.}

Definition. A right $S$-system is a set $M$ and a mapping $(m, a) \rightarrow m a$ of $M \times S$ into $M$ such that $(m a) b=m(a b)$ for each $m \in M, a, b \in S$. A right congruence on $M$ is an equivalence relation $\sigma$ on $M$ such that $m \sigma n$ implies $m a \sigma n a$ for each $m, n \in M, a \in S$. If $m \in M$ then $m_{\sigma}=\{n: n \in$ $M, m \sigma n\} . M / \sigma$ is the set of all congruence classes of $M$ with respect to $\sigma$. Under the composition $m_{\sigma} a=(m a)_{\sigma}$, where $m \in M$ and $a \in S, M / \sigma$ becomes a right $S$-system.

Definition. Let $M$ be a right $S$-system. An $S$-subsystem $L$ of $M$ is trivial if either $L=M$ or $|L|=1$ where $|L|$ is the cardinal number of $L$.

Let $F M=\{m: m \in M, m s=m$ for each $s \in S\}$.

The right $S$-system $M$ is irreducible provided that

(a) $M S \underline{\Phi} F M$ and

(b) $M$ has no nontrivial $S$-subsystem.

One should observe that if $F M \neq \varnothing$, it is an $S$-subsystem of $M$. By (a) 
above, $F M \neq M$. Since $M$ has no nontrivial $S$-subsystem, then $|F M|=1$ and $F M$ is called the zero element of $M$. Furthermore, by (a) above, $|M| \geqslant 2$. By Theorem 5.2 of [4], if $m \in M$ and $m \notin F M$ then $m S=M$.

Definition. If $M$ is a right $S$-system let $M^{\circ}=\{a: a \in S$, every equation $m a b=m$ where $m \in M$ and $b \in S^{\mathbf{1}}$ implies $\left.m \in F M\right\} . M^{\circ}$ is either void or is a two-sided ideal of $S$. It has been proved in [5] and [9] that

$$
N_{L}(S)=\bigcap\left\{M^{\circ}: M \text { is an irreducible right } S \text {-system }\right\} .
$$

The right nil radical congruence will be defined as in [4]. Therefore, let $I_{R}=\{M: M$ is an irreducible right $S$-system $\}$. If $M \in I_{R}, a, b \in S$ let $a \delta_{M} b$ if and only if $m a=m b$ for each $m \in M$. Let $\operatorname{rad}_{R} S=\bigcap_{M \in I_{R}} \delta_{M}$. If $S$ has no irreducible right $S$-system, then $\operatorname{rad}_{R} S$ is the universal relation.

Similarly, let $I_{L}=\{M: M$ is an irreducible left $S$-system $\}$. If $M \in I_{L}$, $a, b \in S$, let $a \delta_{M} b$ if and only if $a m=b m$ for each $m \in M$. Let $\operatorname{rad}_{L} S=$ $\bigcap_{M \in I_{L}} \delta_{M}$. If $S$ has no irreducible left $S$-system, then $\operatorname{rad}_{L} S$ is the universal relation.

The preceding definition is the one used by Hoehnke. However, another characterization of the right nil radical congruence is used more frequently. Thus we have the following.

DEFinition. The right congruence $\sigma$ on $S$ is modular if there exists an element $e$ in $S$ such that es $\sigma s$ for each $s$ in $S$. The element $e$ is a left identity modulo $\sigma$.

LEMMA 4.1. $\operatorname{rad}_{R} S=\bigcap(\sigma: \sigma$ is a modular right congruence on $S$ such that $S / \sigma$ is irreducible).

Proof. See Corollary 6.15 of [4].

We shall now consider a semigroup $S$ such that $\operatorname{rad}_{R} S \neq \operatorname{rad}_{L} S$.

Let $S$ be a set containing at least three elements. Let $\left(S,{ }^{\circ}\right)$ be the left zero semigroup on $S$. Thus, if $x, y \in S$ then $x \circ y=x$. If $M$ is a right $S$. system then $M S \subseteq F M$. Thus $S$ has no irreducible right $S$-system and $\operatorname{rad}_{R} S$ is the universal relation. However, $\operatorname{rad}_{L} S$ is the identity relation.

Throughout the remainder of this paper, we shall use notation found in [2]. Thus $M^{\circ}(G, I, \Lambda, P)$ will be a regular Rees $I \times \Lambda$ matrix semigroup over a group with zero $G^{\circ}$, with sandwich matrix $P=\left(p_{\lambda i}\right)$. Since $M^{\circ}$ is regular, by Lemma 3.1 of [2], each row and each column of $P$ contains a nonzero entry. The identity element of $G$ will be denoted by $e$.

The right nil radical congruence of $M^{\circ}$ will now be studied. Throughout this section let $\sigma$ be a modular right congruence on $M^{\circ}$ with left identity $x$ such that $M^{\circ} / \sigma$ is irreducible. If $y \in M^{\circ}$, let 
$K_{y}=\left\{(i, \lambda):(i, \lambda) \in I \times \Lambda\right.$, there exists $(a)_{i \lambda} \in M^{\circ}$ such that $\left.(a)_{i \lambda} \sigma y\right\}$.

Lemma 4.2. If $(m, \omega) \in K_{x}$ then $p_{\omega m} \neq 0$ and $\left(p_{\omega m}^{-1}\right)_{m \omega} \sigma x$.

Proof. Suppose that $(m, \omega) \in K_{x}$. Let $b \in G$ such that $(b)_{m \omega} \sigma x$. Since $(b)_{m \omega}$ is a left identity modulo $\sigma$, then $\left(b p_{\omega m} b\right)_{m \omega}=(b)_{m \omega}^{2} \sigma(b)_{m \omega}$. Thus $b p_{\omega m} b \neq 0$, which implies that $p_{\omega m} \neq 0$. Furthermore,

$$
\left(p_{\omega m}^{-1}\right)_{m \omega} \sigma(b)_{m \omega}\left(p_{\omega m}^{-1}\right)_{m \omega}=(b)_{m \omega} \sigma x .
$$

Definition. Let $(m, \omega) \in K_{x}$ and let $H=\left\{h: h \in G,\left(h p_{\omega m}^{-1}\right)_{m \omega} \sigma x\right\}$.

LEMMA 4.3. $H$ is a subgroup of $G$.

Proof. By Lemma 4.2, $e \in H$ and thus $H$ is nonempty. Suppose that $a$ is an element of $H$. Thus $\left(a p_{\omega m}^{-1}\right)_{m \omega}$ is a left identity modulo $\sigma$. Therefore

$$
x \sigma\left(e p_{\omega m}^{-1}\right)_{m \omega}=\left(a p_{\omega m}^{-1}\right)_{m \omega}\left(a^{-1} p_{\omega m}^{-1}\right)_{m \omega} \sigma\left(a^{-1} p_{\omega m}^{-1}\right)_{m \omega} .
$$

Thus $a^{-1} \in H$.

Now suppose that each of $a$ and $b$ is in $H$. Thus

$$
\left(a b p_{\omega m}^{-1}\right)_{m \omega}=\left(a p_{\omega m}^{-1}\right)_{m \omega}\left(b p_{\omega m}^{-1}\right)_{m \omega} \sigma\left(b p_{\omega m}^{-1}\right)_{m \omega} \sigma x,
$$

which implies $a b \in H$. Thus $H$ is a subgroup of $G$.

LEMMA 4.4. Suppose that $a$ is an element of $G$ such that $(a)_{i \pi} \sigma 0$ for some $\pi \in \Lambda$. Then $(b)_{i \lambda} \sigma 0$ for each $b$ in $G^{\circ}$ and each $\lambda$ in $\Lambda$.

Proof. Since $P$ is regular, let $j$ be an element of $I$ such that $p_{\pi j} \neq 0$. Therefore

$$
(b)_{i \lambda}=(a)_{i \pi}\left(p_{\pi j}^{-1} a^{-1} b\right)_{j \lambda} \sigma 0\left(p_{\pi j}^{-1} a^{-1} b\right)_{j \lambda}=0 \text {. }
$$

Definition. If $(b)_{i \lambda} \sigma 0$ for each $b \in G^{\circ}$ and each $\lambda \in \Lambda$ then we shall say that $i$ is a $\sigma$-zero row. If this is not the case then we shall say that $i$ is a o-nonzero row.

If $\mu, \lambda \in \Lambda$ then $\mu$ and $\lambda$ are row-compatible provided that, for each $i \in I, p_{\mu i} \neq 0$ if and only if $p_{\lambda i} \neq 0$. Similarly, if $i, j \in I$ then $i$ and $j$ are column-compatible provided that, for each $\lambda \in \Lambda, p_{\lambda i} \neq 0$ if and only if $p_{\lambda j} \neq 0$.

LEMma 4.5. Row $i$ is a o-nonzero row if and only if $p_{\omega i} \neq 0$. Furthermore, if $(a)_{i \mu} \& 0$ and $(b)_{j \lambda} \in M^{\circ}$ such that $(a)_{i \mu} \sigma(b)_{j \lambda}$ then $\mu$ and $\lambda$ are row-compatible. 
Proof. Suppose that $i$ is a $\sigma$-nonzero row. Let $(a)_{i \pi} \in M^{\circ}$ such that $(a)_{i \pi} \phi 0$. Since $\left(p_{\omega m}^{-1}\right)_{m \omega}$ is a left identity modulo $\sigma$, then

$$
\left(p_{\omega m}^{-1} p_{\omega i} a\right)_{m \pi}=\left(p_{\omega m}^{-1}\right)_{m \omega}(a)_{i \pi} \sigma(a)_{i \pi} \not 0 .
$$

Thus $p_{\omega m}^{-1} p_{\omega i} a \neq 0$, which implies $p_{\omega i} \neq 0$.

Conversely, suppose that $i$ is a $\sigma$-zero row. Let $b \in G, \lambda \in \Lambda$, such that $(b)_{i \lambda} \sigma 0$. Thus

$$
\left(p_{\omega m}^{-1} p_{\omega i} b\right)_{m \lambda}=\left(p_{\omega m}^{-1}\right)_{m \omega}(b)_{i \lambda} \sigma(b)_{i \lambda} \sigma 0 .
$$

By Lemma 4.4 , since $m$ is a $\sigma$-nonzero row, then $p_{\omega m}^{-1} p_{\omega i} b=0$. Since $p_{\omega m}^{-1}$, $b \in G$ this implies $p_{\omega i}=0$.

Now suppose that $(a)_{i \mu} \phi 0$ and $(b)_{j \lambda} \sigma(a)_{i \mu}$. We wish to show that $\mu$ and $\lambda$ are row-compatible. Let $k \in I$ such that $p_{\mu k} \neq 0$. Since $(a)_{i \mu} \sigma(b)_{j \lambda}$ then

$$
\begin{aligned}
\left(p_{\omega m}^{-1} p_{\omega j} b p_{\lambda k} p_{\mu k}^{-1} a^{-1} p_{\omega i}^{-1} p_{\omega m} p_{\omega m}^{-1}\right)_{m \omega} \\
\quad=\left(p_{\omega m}^{-1}\right)_{m \omega}(b)_{j \lambda}\left(p_{\mu k}^{-1} a^{-1} p_{\omega i}^{-1}\right)_{k \omega} \sigma\left(p_{\omega m}^{-1}\right)_{m \omega}(a)_{i \mu}\left(p_{\mu k}^{-1} a^{-1} p_{\omega i}^{-1}\right)_{k \omega} \\
\quad=\left(p_{\omega m}^{-1} p_{\omega i} a p_{\mu k} p_{\mu k}^{-1} a^{-1} p_{\omega i}^{-1}\right)_{m \omega}=\left(p_{\omega m}^{-1}\right)_{m \omega} .
\end{aligned}
$$

Thus $p_{\omega m}^{-1} p_{\omega j} b p_{\lambda k} p_{\mu k}^{-1} a^{-1} p_{\omega i}^{-1} p_{\omega m} \in H$. Therefore $p_{\lambda k} \neq 0$. Similarly, if $p_{\lambda k} \neq 0$ then $p_{\mu k} \neq 0$. Thus $\mu$ and $\lambda$ are row-compatible.

Lemma 4.6. Let $(a)_{i \mu} \& 0$ and let $j$ be a $\sigma$-nonzero row. Then $(j, \mu)$ $\in K_{(a)_{i \mu}}$.

Proof. Since $j$ is a $\sigma$-nonzero row then, by Lemma $4.5, p_{\omega j} \neq 0$. By Lemma 4.4, $\left(p_{\omega j}^{-1}\right)_{j \omega} \not 0$. Since $M^{\circ} / \sigma$ is irreducible, there exists $(c)_{k \lambda} \in M^{\circ}$ such that $\left(p_{\omega j}^{-1}\right)_{j \omega}(c)_{k \lambda} \sigma x$. Thus $(j, \lambda) \in K_{x}$ and, by Lemma 4.2, $\left(p_{\lambda j}^{-1}\right)_{j \lambda}$ is a left identity modulo $\sigma$. Therefore

$$
\left(p_{\lambda j}^{-1} p_{\lambda i} a\right)_{j \mu}=\left(p_{\lambda j}^{-1}\right)_{j \lambda}(a)_{i \mu} \sigma(a)_{i \mu} .
$$

Thus $(j, \mu) \in K_{(a)_{i \mu}}$.

Lemma 4.7. Let $\left[(a)_{i \mu}\right]_{\sigma}$ be a nonzero element of $\mu^{\circ} / \sigma$. Then

$$
\begin{array}{r}
{\left[(a)_{i \mu}\right]_{\sigma}=\left\{\left(p_{\omega j}^{-1} p_{\omega m} h p_{\omega m}^{-1} p_{\omega i} a p_{\mu k} p_{\lambda k}^{-1}\right)_{j \lambda}: h \in H,(j, \lambda) \in K_{(a)_{i \mu}},\right.} \\
\left.k \in I \text { such that } p_{\mu k} \neq 0\right\} .
\end{array}
$$

Proof. Let $(b)_{j \lambda} \in\left[(a)_{i \mu}\right]_{\sigma}$ and $k \in I$ such that $p_{\mu k} \neq 0$. Since $(b)_{j \lambda} \sigma(a)_{i \mu}$ then $\mu$ and $\lambda$ are row-compatible. Furthermore, as was seen in 
the proof of Lemma 4.5, $p_{\omega m}^{-1} p_{\omega j} b p_{\lambda k} p_{\mu k}^{-1} a^{-1} p_{\omega i}^{-1} p_{\omega m} \in H$. Thus $b \in$ $p_{\omega i}^{-1} p_{\omega m} H p_{\omega m}^{-1} p_{\omega i} a p_{\mu k} p_{\lambda k}^{-1}$.

Conversely, let $h^{\prime} \in H,(j, \lambda) \in K_{(a)_{i \mu}}$, and $k \in I$ such that $p_{\mu k} \neq 0$. Since $(j, \lambda) \in K_{(a)_{i \mu}}$, there exists $b \in G$ such that $(b)_{j \lambda} \sigma(a)_{i \mu}$. By Lemma 4.5 and the work above, $p_{\omega j} \neq 0, p_{\lambda k} \neq 0$ and there exists $\bar{h} \in H$ such that $b=p_{\omega j}^{-1} p_{\omega m} \bar{h} p_{\omega m}^{-1} p_{\omega i} a p_{\mu k} p_{\lambda k}^{-1}$. But, for each $h \in H$,

$$
\begin{gathered}
\left(p_{\omega j}^{-1} p_{\omega m} h p_{\omega m}^{-1} p_{\omega t} a p_{\mu k} p_{\lambda k}^{-1}\right)_{j \lambda} \sigma\left(p_{\omega m}^{-1}\right)_{m \omega}\left(p_{\omega j}^{-1} p_{\omega m} h p_{\omega m}^{-1} p_{\omega l} a p_{\mu k} p_{\lambda k}^{-1}\right)_{j \lambda} \\
=\left(h p_{\omega m}^{-1} p_{\omega i} a p_{\mu k} p_{\lambda k}^{-1}\right)_{m \lambda}=\left(h p_{\omega m}^{-1}\right)_{m \omega}\left(a p_{\mu k} p_{\lambda k}^{-1}\right)_{i \lambda} \sigma\left(a p_{\mu k} p_{\lambda k}^{-1}\right)_{i \lambda} .
\end{gathered}
$$

Thus

$$
\begin{gathered}
\left(p_{\omega j}^{-1} p_{\omega m} h^{\prime} p_{\omega m}^{-1} p_{\omega l} a p_{\mu k} p_{\lambda k}^{-1}\right)_{j \lambda} \sigma\left(p_{\omega j}^{-1} p_{\omega m} \bar{h} p_{\omega m}^{-1} p_{\omega i} a p_{\mu k} p_{\lambda k}^{-1}\right)_{j \lambda} \\
=(b)_{j \lambda} \sigma(a)_{i \mu} .
\end{gathered}
$$

Therefore

$$
\begin{aligned}
& \left\{\left(p_{\omega j}^{-1} p_{\omega m} h p_{\omega m}^{-1} p_{\omega l} a p_{\mu k} p_{\lambda k}^{-1}\right)_{j \lambda}: h \in H,(j, \lambda) \in K_{(a)_{i \mu}}\right. \\
& \left.k \in I \text { such that } p_{\mu k} \neq 0\right\} \subseteq\left[(a)_{i \mu}\right]_{\sigma} .
\end{aligned}
$$

LEMMA 4.8. Let $H$ be a subgroup of $G$ and let $(m, \omega) \in I \times \Lambda$ such that $p_{\omega m} \neq 0$. Let $H_{m \omega}$ be a right congruence on $M^{\circ}$ defined as follows: $z=\left\{(a)_{i \mu}: a \in G, p_{\omega i}=0\right\} \cup\{0\}$, if $(a)_{i \mu},(b)_{j \lambda} \notin z$ then $(a)_{i \mu} H_{m \omega}(b)_{j \lambda}$ if and only if $\mu=\lambda, p_{\omega m}^{-1} p_{\omega j} b a^{-1} p_{\omega i}^{-1} p_{\omega m} \in H$. Then $H_{m \omega}$ is a modular right congruence such that $M^{\circ} / H_{m \omega}$ is irreducible with zero element $z$.

Proof. Clearly $H_{m \omega}$ is reflexive. Since $p_{\omega m}^{-1} p_{\omega j} b a^{-1} p_{\omega i}^{-1} p_{\omega m} \in H$ if and only if $p_{\omega m}^{-1} p_{\omega i} a b^{-1} p_{\omega j}^{-1} p_{\omega m} \in H$, then $H_{m \omega}$ is symmetric.

To show that $H_{m \omega}$ is transitive let $(a)_{i \mu},(b)_{j \mu},(c)_{k \mu} \notin z$ such that $(a)_{i \mu} H_{m \omega}(b)_{j \mu}$ and $(b)_{j \mu} H_{m \omega}(c)_{k \mu}$. Since $p_{\omega m}^{-1} p_{\omega i} a b^{-1} p_{\omega j}^{-1} p_{\omega m} \in H$ and $p_{\omega m}^{-1} p_{\omega j} b c^{-1} p_{\omega k}^{-1} p_{\omega m} \in H$ then $p_{\omega m}^{-1} p_{\omega i} a c^{-1} p_{\omega k}^{-1} p_{\omega m} \in H$. Therefore $(a)_{i \mu} H_{m \omega}(c)_{k \mu}$ and $H_{m \omega}$ is transitive.

To show that $H_{m \omega}$ is a right congruence let $(a)_{i \mu},(b)_{j \lambda},(c)_{k \pi} \in M^{\circ}$ such that $(a)_{i \mu} H_{m \omega}(b)_{j \lambda}$. If $(a)_{i \mu},(b)_{j \lambda} \in z$ then $(a)_{i \mu}(c)_{k \pi},(b)_{j \lambda}(c)_{k \pi} \in z$, which implies $(a)_{i \mu}(c)_{k \pi} H_{m \omega}(b)_{j \lambda}(c)_{k \pi}$. If $(a)_{i \mu},(b)_{j \lambda} \notin z$ then $\mu=\lambda$ and $p_{\omega m}^{-1} p_{\omega j} b a^{-1} p_{\omega i}^{-1} p_{\omega m} \in H$. Therefore

$$
p_{\omega m}^{-1} p_{\omega j}\left(b p_{\mu k} c\right)\left(a p_{\mu k} c\right)^{-1} p_{\omega i}^{-1} p_{\omega m} \in H,
$$

which implies $(a)_{i \mu}(c)_{k \pi} H_{m \omega}(b)_{j \lambda}(c)_{k \pi}$. Thus $H_{m \omega}$ is a right congruence on $M^{\circ}$. 
It is clear that $\left(p_{\omega m}^{-1}\right)_{m \omega}$ is a left identity modulo $H_{m \omega}$. To show that $M^{\circ} / H_{m \omega}$ is irreducible, let $(a)_{i \mu} \notin z$ and $(b)_{j \lambda} \in M^{\circ}$. We must find $(c)_{k \pi} \in$ $M^{\circ}$ such that $(a)_{i \mu}(c)_{k \pi} H_{m \omega}(b)_{j \lambda}$. If $(b)_{j \lambda} \in z$ let $(c)_{k \pi}=0$. If $(b)_{j \lambda} \notin z$ let $k$ be an element of $I$ such that $p_{\mu k} \neq 0$ and let $(c)_{k \pi}=\left(p_{\mu k}^{-1} a^{-1} p_{\omega i}^{-1} p_{\omega j} b\right)_{k \lambda}$. Thus $\left[(a)_{i \mu}\right]_{H_{m \omega}} M^{\circ}=M^{\circ} / H_{m \omega}$ for any $(a)_{i \mu} \notin z$, which implies that $M^{\circ} / H_{m \omega}$ is irreducible.

Lemma 4.9. $\operatorname{rad}_{R} M^{\circ}=\bigcap\left(H_{m \omega}: H\right.$ is a subgroup of $\left.G, p_{\omega m} \neq 0\right)$.

Proof. By Lemmas 4.1 and 4.8,

$$
\operatorname{rad}_{R} M^{\circ} \subseteq \bigcap\left(H_{m \omega}: H \text { is a subgroup of } G, p_{\omega m} \neq 0\right) .
$$

Conversely, let $\sigma$ be a modular right congruence on $M^{\circ}$ such that $M^{\circ} \%$ is irreducible. By Lemma 4.2, there exists $(m, \omega) \in I \times \Lambda$ such that $\left(p_{\omega m}^{-1}\right)_{m \omega}$ is a left identity modulo $\sigma$. By Lemmas 4.4 and $4.5, z=\left\{(a)_{i \mu}: a \in G, p_{\omega i}=\right.$ $0\} \cup\{0\}$ is the zero element of $M^{\circ} / \sigma$.

Let $H=\left\{h: h \in G,\left(h p_{\omega m}^{-1}\right)_{m \omega}\right.$ is a left identity modulo $\left.\sigma\right\}$. By Lemma 4.3, $H$ is a subgroup of $G$. We wish to show that $H_{m \omega} \subseteq \sigma$. Let $(a)_{i \mu},(b)_{j \lambda}$ $\notin z$ such that $(a)_{i \mu} H_{m \omega}(b)_{j \lambda}$. Thus $\mu=\lambda$ and $p_{\omega m}^{-1} p_{\omega j} b a^{-1} p_{\omega i}^{-1} p_{\omega m} \in H$. Furthermore, by Lemma 4.6, $(j, \mu) \in K_{(a)_{i \mu}}$. Let $h \in H$ such that $b=$ $p_{\omega j}^{-1} p_{\omega m} h p_{\omega m}^{-1} p_{\omega i} a$ and let $k \in I$ such that $p_{\mu k} \neq 0$. Since

$$
(b)_{j \lambda}=\left(p_{\omega j}^{-1} p_{\omega m} h p_{\omega m}^{-1} p_{\omega i} a p_{\mu k} p_{\mu k}^{-1}\right)_{j \mu}
$$

then, by Lemma 4.7, $(b)_{j \lambda} \sigma(a)_{i \mu}$. Thus $H_{m \omega} \subseteq \sigma$.

Therefore, by Lemma 4.1,

$$
\bigcap\left(H_{m \omega}: H \text { is a subgroup of } G, p_{\omega m} \neq 0\right) \subseteq \operatorname{rad}_{R} M^{\circ} .
$$

THEOREM 4.10. $(a)_{i \lambda} \operatorname{rad}_{R} M^{\circ}(b)_{j \mu}$ if and only if $\lambda=\mu$ and $p_{\omega i} a=$ $p_{\omega j} b$ for each $\omega \in \Lambda$.

Proof. Suppose that $(a)_{i \lambda} \operatorname{rad}_{R} M^{\circ}(b)_{j \mu}$. Since $P$ is regular, let $\omega \in \Lambda$ such that $p_{\omega i} \neq 0$. Let $H=\{e\}$. Since $(a)_{i \lambda} H_{i \omega}(b)_{j \mu}$ and $p_{\omega i} \neq 0$ then $\lambda=\mu, p_{\omega j} \neq 0$, and $p_{\omega i}^{-1} p_{\omega j} b a^{-1} p_{\omega i}^{-1} p_{\omega i} \in H=\{e\}$. Thus $p_{\omega j} b=p_{\omega i} a$. Now suppose that $\gamma \in \Lambda$ such that $p_{\gamma i}=0$. Let $k \in I$ such that $p_{\gamma k} \neq 0$. Thus $(a)_{i \lambda}$ and $(b)_{j \mu}$ are elements of $z$, the zero element of $M^{\circ} / H_{k \gamma}$. Thus $p_{\gamma j}=0$ also and $p_{\gamma i} a=0=p_{\gamma j} b$.

Conversely, suppose that $\lambda=\mu$ and $p_{\omega i} a=p_{\omega j} b$ for each $\omega \in \Lambda$. Let $(m, \omega) \in I \times \Lambda$ such that $p_{\omega m} \neq 0$ and let $H$ be a subgroup of $G$. If $0=$ $p_{\omega i} a$ then either $p_{\omega i}=0$ or $a=0$. In either case, $(a)_{i \lambda} \in z$, the zero element of $M^{\circ} / H_{m \omega}$. Since $0=p_{\omega i} a=p_{\omega j} b$ then $(b)_{j \lambda} \in z$ also. Thus $(a)_{i \lambda} H_{m \omega}(b)_{i \lambda}$. 
On the other hand, if $0 \neq p_{\omega i} a$ then $p_{\omega i} \neq 0, a \neq 0, b \neq 0$, and $p_{\omega j} \neq 0$. Furthermore,

$$
p_{\omega m}^{-1}\left(p_{\omega j} b\right) a^{-1} p_{\omega i}^{-1} p_{\omega m}=p_{\omega m}^{-1}\left(p_{\omega i} a\right) a^{-1} p_{\omega i}^{-1} p_{\omega m}=e \in H .
$$

Thus $(a)_{i \lambda} H_{m \omega}(b)_{j \lambda}$. Since this is true for each $H_{m \omega}$, then $(a)_{i \lambda} \operatorname{rad}_{R} M^{\circ}$ $(b)_{j \lambda}$.

Definition (GreEn'S Relation). If $a, b \in S$ then we define $a L b$ to mean that $a$ and $b$ generate the same principal left ideal of $S$.

Corollary 4.11. $\operatorname{rad}_{R} M^{\circ} \subseteq L$.

Proof. Suppose that $(a)_{i \lambda} \operatorname{rad}_{R} M^{\circ}(b)_{j \mu}$. Let $\omega \in \Lambda$ such that $p_{\omega i} \neq$ 0. Thus $\lambda=\mu$ and $(a)_{i \lambda}=\left(p_{\omega i}^{-1} p_{\omega j} b\right)_{i \lambda}=\left(p_{\omega i}^{-1}\right)_{i \omega}(b)_{j \mu} \in M^{\circ}(b)_{j \mu}$. Therefore $(a)_{i \lambda} \cup M^{\circ}(a)_{i \lambda} \subseteq(b)_{j \mu} \cup M^{\circ}(b)_{j \mu}$. Similarly, $(b)_{j \mu} \cup M^{\circ}(b)_{j \mu} \subseteq(a)_{i \lambda} \cup M^{\circ}(a)_{i \lambda}$.

COROLlary 4.12. $\operatorname{rad}_{R} M^{\circ}=\operatorname{rad}_{L} M^{\circ}$ if and only if they are both the identity relation.

Proof. Suppose that $\operatorname{rad}_{R} M^{\circ}=\operatorname{rad}_{L} M^{\circ}$. Let $(a)_{i \lambda} \operatorname{rad}_{R} M^{\circ}(b)_{j \mu}$. By Theorem 4.10 and its dual, $\lambda=\mu$ and $i=j$. Furthermore, $p_{\omega i} a=p_{\omega i} b$ for each $\omega \in \Lambda$ implies that $a=b$. Thus $\operatorname{rad}_{R} M^{\circ}=\operatorname{rad}_{L} M^{\circ}=$ the identity relation.

It will be shown later that the Jacobson radical congruence of $M^{\circ}$ does not depend upon the subgroups of $G$ but merely upon the positions of the nonzero entries of $P$.

5. The Jacobson radical congruence.

Definition. A right $S$-system $M$ is totally irreducible provided that

(a) $M S \nsubseteq \subseteq F M$ and

(b) $M$ has no nontrivial homomorphisms.

One should observe that every totally irreducible right $S$-system is irreducible.

Definition. Let $T_{R}=\{M: M$ is a totally irreducible right $S$-system $\}$. If $M \in T_{R}, a, b \in S$ let $a \delta_{M} b$ if and only if $m a=m b$ for each $m \in M$. Let $\overline{\operatorname{rad}}_{R} S=\bigcap_{M \in T_{R}} \delta_{M}$. If $T_{R}=\varnothing$, then $\overline{\operatorname{rad}}_{R} S$ is the universal relation. $\overline{\operatorname{rad}}_{R} S$ is the right Jacobson radical congruence on $S$.

Similarly define $\overline{\operatorname{rad}}_{L} S$, the left Jacobson radical congruence on $S$.

DEFINITION. If $e \in S$ then $e$ is a right quasi-regular element of $S$ if the only right congruence on $S$ which has $e$ as a left identity is the universal relation. An ideal of $S$ is quasi-regular if each element contained in it is a right quasi-regular element of $S$.

THEOREM 5.1. If $e$ is an element of $S$ such that for each integer $k>1$, 
$e^{k}$ is right quasi-regular then $0_{L}(S) \neq \varnothing$ and $e \in N_{L}(S)$.

Proof. See [9, Lemma 1.3].

A result similar to that found for the nil radical will now be proved. $M^{\circ}$ will be defined as before; thus

$$
M^{\circ}=\left\{a: a \in S \text {, every equation } m a b=m \text { where } m \in M \text { and } b \in S^{1}\right.
$$

implies $m \in F M$ \}.

If $T_{R}=\varnothing$ then we shall say that $\bigcap_{M \in T_{R}} M^{\circ}=S$.

THEOREM 5.2. If $N_{L}(S) \neq \varnothing$, then $N_{L}(S)=\bigcap_{M \in T_{R}} M^{\circ}$.

Proof. Since $N_{L}(S)$ is nonvoid let $a \in N_{L}(S)$. Let $M \in T_{R}$ and let $m \in M, b \in S^{1}$ such that $m a b=m$. Since $a b \in N_{L}(S)$ let $p$ be a positive integer such that $(a b)^{p} \in 0_{L}(S)$. Since $m S=m(a b)^{p} S=m(a b)^{p}=m$ then $m \in F M$. Thus $N_{L}(S) \subseteq \bigcap_{M \in T_{R}} M^{\circ}$.

It will now be shown that $\bigcap_{M \in T_{R}} M^{\circ}$ is a quasi-regular ideal of $S$. Let $c \in \bigcap_{M \in T_{R}} M^{\circ}$ and $s \in S$. It must be shown that $s c, c s \in \bigcap_{M \in T_{R}} M^{\circ}$. Let $M \in T_{R}$ and $m \in M$ such that $m c s b=m$ for some $b \in S^{1}$. Since $c \in M^{\circ}$ then $m \in F M$. Thus $c s \in \bigcap_{M \in T_{R}} M^{\circ}$. Now suppose that $M \in T_{R}, m \in M$ such that $m s c b=m$ for some $b \in S^{1}$. Thus $m s c b s=m s$. Since $c \in M^{\circ}$ then $m s \in F M$. Therefore

$$
m s=m s(c b s c b)=m(s c b)^{2}=m .
$$

Since $m s \in F M$ then $m \in F M$. Therefore $s c \in \bigcap_{M \in T_{R}} M^{\circ}$ and $\bigcap_{M \in T_{R}} M^{\circ}$ is an ideal of $S$.

Suppose that $e$ is an element of $\bigcap_{M \in T_{R}} M^{\circ}$ which is not right quasiregular. Thus there exists a right congruence $\tau$ on $S$ such that $\tau$ is not the universal relation and $e$ is a left identity modulo $\tau$. By Theorem 1 of [10], let $\sigma$ be a maximal right congruence on $S$ which contains $\tau$. Thus $\sigma$ is a modular maximal right congruence on $S$ and $e$ is a left identity modulo $\sigma$. Therefore, by Theorem 6.2 of [4], $S / \sigma \in T_{R}$. But $e_{\sigma} e=e_{\sigma}$ where $e_{\sigma} \notin$ $F(S / \sigma)$. This contradicts the fact that $e \in(S / \sigma)^{\circ}$. Thus $\bigcap_{M \in T_{R}} M^{\circ}$ is a quasi-regular ideal of $S$. By Theorem 5.1 above, $\bigcap_{M \in T_{R}} M^{\circ} \subseteq N_{L}(S)$.

Lemma 5.3. $\overline{\operatorname{rad}}_{R} S=\bigcap_{\sigma \in \Sigma_{S}} \sigma$ where

$$
\Sigma_{S}=\{\sigma: \sigma \text { is a modular maximal right congruence on } S\} \text {. }
$$

Proof. See Corollary 6.16 of [4].

Lemma 5.4. Let $S$ be a semigroup such that $N_{L}(S) \neq \varnothing$. The ideal 
$I$ of $S$ is nil if and only if $I / \sigma \subseteq F(S / \sigma)$ for each $\sigma \in \Sigma_{S}$.

Proof. Suppose that $\sigma \in \Sigma_{S}$ such that $N_{L}(S) / \sigma \underline{\Phi} F(S / \sigma)$. Let $x \in$ $N_{L}(S)$ such that $x_{\sigma} \notin F(S / \sigma)$. By Theorem 6.2 of [4], $S / \sigma \subseteq T_{R}$. Since $x_{\sigma} \notin$ $F(S / \sigma)$ and $S / \sigma$ is irreducible then $x_{\sigma} S=S / \sigma$. Let $e$ be a left identity modulo $\sigma$ and let $s \in S$ such that $x_{\sigma} s=e_{\sigma}$. Since $x s \in N_{L}(S)$, let $p$ be a positive integer such that $(x s)^{p} \in 0_{L}(S)$. Thus $(x s)_{\sigma}^{p}=(x s)_{\sigma}^{p} S=e_{\sigma} S=S / \sigma$, which contradicts the fact that $\sigma$ is not the universal relation on $S$. Therefore, for each $\sigma \in \Sigma_{S}, N_{L}(S) / \sigma \subseteq F(S / \sigma)$. Thus if $I$ is a nil ideal of $S$ then $I / \sigma \in F(S / \sigma)$ for each $\sigma \in \Sigma_{S}$.

Conversely, suppose that $I$ is an ideal of $S$ which is not nil. By Theorem 5.2 , let $M \in T_{R}$ such that $I \underline{\Phi} M^{\circ}$. By Theorem 6.2 of [4], let $\sigma \in \Sigma_{S}$ such that $M \cong S / \sigma$. Since $I \nsubseteq M^{\circ}$, let $m \in M, i \in I, b \in S^{1}$ such that $m i b=m$ and $m \notin F M$. Since $M \cong S / \sigma$, there exists $s \in S$ such that $s_{\sigma} i b=s_{\sigma}$ and $s_{\sigma} \notin F(S / \sigma)$. Thus $s i b \in I$ such that $(s i b)_{\sigma} \notin F(S / \sigma)$.

Lemma 5.5. Let $\sigma \in \Sigma_{S}$ and $T$ be a right ideal of $S$ such that $T / \sigma \underline{\Xi}$ $F(S / \sigma)$. Then $T$ intersects each element of $S / \sigma$.

Proof. Let $t \in T$ such that $t_{\sigma} \notin F(S / \sigma)$. Since $S / \sigma$ is an irreducible right $S$-system then $t_{\sigma} S=S / \sigma$. Thus if $x_{\sigma} \in S / \sigma$ there exists $s \in S$ such that $t_{\sigma} s=x_{\sigma}$. Thus $t s \in x_{\sigma} \cap T$ and $T$ intersects each element of $S / \sigma$.

Notation. Let $T$ be a subsemigroup of $S$ and let $\sigma$ be a right congruence on $S$. Then $\sigma \mid T$ will denote $\sigma$ restricted to $T$. Thus $\sigma \mid T$ is a right congruence on $T$ defined as follows: If $t, u \in T$ then $t \sigma \mid T u$ if and only if $t \sigma u$.

THEOREM 5.6. Let $S$ be a semigroup such that $N_{L}(S) \neq \varnothing$. If $T$ is an ideal of $S$ then $\overline{\operatorname{rad}}_{R} T=\overline{\operatorname{rad}}_{R} S \mid T$.

Proof. First suppose that $T \subseteq N_{L}(T)$. It will now be shown that $T$ has no totally irreducible right $T$-system. Suppose that $M$ is a totally irreducible right $T$-system and $m \in M$ such that $m \notin F M$. Since $M$ is irreducible then $m T=M$. Let $t$ be an element of $T$ such that $m t=m$. By Theorem 5.2, $t \in T \subseteq N_{L}(T) \subseteq M^{\circ}$. Thus $m t=m$ implies $m \in F M$, which is a contradiction. Therefore $T$ has no totally irreducible right $T$-system and $\overline{\operatorname{rad}}_{R} T$ is the universal relation on $T$.

If $\overline{\operatorname{rad}}_{R} S$ is the universal relation on $S$ then $\overline{\operatorname{rad}}_{R} T=\overline{\operatorname{rad}}_{R} S \mid T$. If $\overline{\operatorname{rad}}_{R} S$ is not the universal relation on $S$, let $\sigma \in \Sigma_{S}$. Suppose that $T / \sigma \nsubseteq \underline{I}$ $F(S / \sigma)$. By Lemma 5.5, let $e$ be an element of $T$ which is a left identity modulo $\sigma$. Since $e \in T \subseteq N_{L}(T)$, let $p$ be a positive integer such that 
$e^{p} \in 0_{L}(T)$. Thus, $S / \sigma=e_{\sigma} S=e_{\sigma}^{p+1} S=e_{\sigma}^{p} e S \subseteq e_{\sigma}^{p} T=e_{\sigma}^{p}$, which contradicts the fact that $\sigma$ is not the universal relation. Thus $T / \sigma \subseteq F(S / \sigma)$. Therefore if $t, u \in T$ then $t_{\sigma}, u_{\sigma} \in F(S / \sigma)$, which implies $t \sigma u$. Thus $\sigma \mid T$ is the universal relation on $T$ for each $\sigma \in \Sigma_{S}$. Therefore $\overline{\operatorname{rad}}_{R} S \mid T=$ the universal relation on $T=\overline{\operatorname{rad}}_{R} T$.

Now suppose that $T \underline{\Phi} N_{L}(T)$. By Theorem 5.2, there exist totally irreducible right $T$-systems. Let $\tau$ be an element of $\Sigma_{T}$ and $e$ be a left identity modulo $\tau$. If $a, b \in S$ let $a \gamma_{\tau} b$ if and only if $a p \tau b p$ for each $p \in T$. It will be shown that $\gamma_{\tau}$ is a right congruence on $S$ with left identity $e$ such that $\tau=\gamma_{\tau} \mid T$.

It is clear that $\gamma_{\tau}$ is an equivalence relation on $S$ with left identity $e$. Let $a, b, s \in S$ such that $a \gamma_{\tau} b$. Since $T$ is an ideal of $S$ then $s p \in T$ for each $p \in T$. Therefore $a(s p) \tau b(s p)$ for each $p$ in $T$. Thus $a s \gamma_{\tau} b s$ and $\boldsymbol{\gamma}_{\tau}$ is a right congruence on $S$.

Let $t, u \in T$ such that $t \tau u$. Since $\tau$ is a right congruence on $T$, then tp $\tau$ up for each $p$ in $T$. Thus $t \gamma_{\tau} u$ and $\tau \subseteq \gamma_{\tau} \mid T$. Let $z$ be an element of $0_{L}(S)$. Therefore $z \in T$ and $(z e)_{\tau}=z_{\tau} \neq e_{\tau}=(e e)_{\tau}$. Thus $z \psi_{\tau} e$ and $\gamma_{\tau} \mid T$ is not the universal relation on $T$. Since $\tau$ is maximal, $\tau=\gamma_{\tau} \mid T$.

It will now be shown that $\gamma_{\tau} \in \Sigma_{S}$. To establish the fact that $\gamma_{\tau}$ is maximal, let $\beta$ be a right congruence on $S$ containing $\gamma_{\tau}$. Either $\beta \mid T$ is the universal relation on $T$ or $\beta \mid T=\tau$. Suppose that $\beta \mid T$ is the universal relation on T. Then $e \beta z$. But $e$ is a left identity modulo $\beta$. Thus if $s \in S, z_{\beta}=z_{\beta} s=$ $e_{\beta} s=s_{\beta}$. Therefore $\beta$ is the universal relation on $S$. Now suppose that $\beta \mid T=$ $\tau$. Let $a, b \in S$ such that $a \beta b$. Thus $e a \beta e b$. Since $e a, e b \in T$ and $\beta \mid T=$ $\tau$ then $e a \tau e b$. Thus ap $\tau$ eap $\tau e b p \tau b p$ for each $p$ in $T$. Therefore $a \gamma_{\tau} b$, which implies $\gamma_{\tau}=\beta$. Therefore $\gamma_{\tau} \in \Sigma_{S}$ and $\gamma_{\tau} \mid T=\tau$. Thus

$$
\overline{\operatorname{rad}}_{R} S\left|T=\bigcap_{\sigma \in \Sigma_{S}} \sigma\right| T \subseteq \bigcap_{\tau \in \Sigma_{T}} \gamma_{\tau} \mid T=\bigcap_{\tau \in \Sigma_{T}} \tau=\overline{\operatorname{rad}}_{R} T .
$$

Conversely, since $T \underline{\Phi} N_{L}(T)$ then $T \underline{\Phi} N_{L}(S)$. By Lemma 5.4, let $\sigma \in$

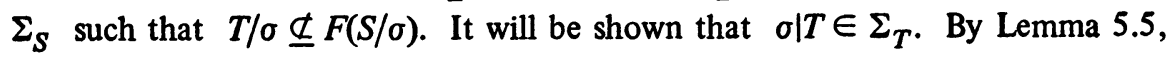
let $e \in T$ such that $e$ is a left identity modulo $\sigma$. Let $\tau$ be a right congru- ence on $T$ such that $\sigma \mid T \subseteq \tau$. Construct $\gamma_{\tau}$ as above, i.e., $a \gamma_{\tau} b$ if and only if $a p \tau b p$ for each $p$ in $T$. Thus $\gamma_{\tau}$ is a right congruence on $S$. To show that $\sigma \subseteq \gamma_{\tau}$ let $a, b \in S$ such that $a \sigma b$. Since $e$ is a left identity modulo $\sigma$, ea $\sigma e b$. Since $e a, e b \in T$ then $e a \sigma \mid T e b$. Thus ap $\sigma \mid T$ eap $\sigma|T e b p \sigma| T b p$ for each $p$ in $T$. But $\sigma \mid T \subseteq \tau$. Thus $a p \tau b p$ for each $p$ in $T$. Therefore $a \gamma_{\tau} b$ and $\sigma \subseteq \gamma_{\tau}$. 
But $\sigma$ is maximal. Thus either $\gamma_{\tau}=\sigma$ or $\gamma_{\tau}$ is the universal relation on $S$. Suppose that $\gamma_{\tau}$ is the universal relation on $S$. Let $z$ be an element of $0_{L}(S)$. Since $z \gamma_{\tau} e$ and $e \in T$ then $z e \tau e e$. Thus $z \tau e$ and, since $e$ is a left identity modulo $\tau, \tau$ is the universal relation on $T$. On the other hand, suppose that $\gamma_{\tau}=\sigma$. Let $t, u \in T$ such that $t \tau u$. Therefore $t p \tau u p$ for each $p \in T$, which implies that $t \gamma_{\tau} u$. But $\gamma_{\tau}=\sigma$; thus $t \sigma \mid T u$. Therefore $\tau \subseteq \sigma \mid T$. Thus $\tau=\sigma \mid T$. Since either $\sigma \mid T=\tau$ or $\tau$ is the universal relation on $T$, then $\sigma \mid T$ is a maximal right congruence on $T$.

Therefore

$$
\overline{\operatorname{rad}}_{R} T=\bigcap_{\tau \in \Sigma_{T}} \tau \subseteq \bigcap\left\{\sigma \mid T: \sigma \in \Sigma_{S}, T / \sigma \subseteq \underline{D}(S / \sigma)\right\}=\bigcap_{\sigma \in \Sigma_{S}} \sigma\left|T=\overline{\operatorname{rad}}_{R} S\right| T .
$$

THEOREM 5.7. Let $S$ be a semigroup such that $N_{L}(S) \neq \varnothing$. Suppose that $T$ is an ideal of $S$ such that $\overline{\operatorname{rad}}_{L} S / T=\overline{\operatorname{rad}}_{R} S / T$ and $\overline{\operatorname{rad}}_{L} T=\overline{\operatorname{rad}}_{R} T$. Then $\overline{\operatorname{rad}}_{L} S=\overline{\operatorname{rad}}_{R} S$.

Proof. Let $A=\left\{\sigma: \sigma \in \Sigma_{S}, T / \sigma \subseteq F(S / \sigma)\right\}$ and $B=\left\{\sigma: \sigma \in \Sigma_{S}\right.$,

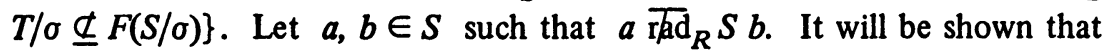
$a \overline{\operatorname{rad}}_{L} S b$. By Lemma 5.3, let $\sigma \in \Sigma_{S}$ such that $a \not b b$.

Suppose that $\sigma \in A$. Let $\sigma^{\prime}$ be a right congruence on $S / T$, the factor semigroup of $S$ modulo $T$, defined as follows: If $x, y \in S$ then $[x] \sigma^{\prime}[y]$ if and only if $x \sigma y$. To show that $\sigma^{\prime}$ is well defined suppose that $[x]=\left[x_{1}\right]$, $[y]=\left[y_{1}\right]$ and $[x] \sigma^{\prime}[y]$. Since $[x]=\left[x_{1}\right]$ then either $x=x_{1}$ or $x, x_{1} \in$ T. Since $T / \sigma \subseteq F(S / \sigma)$, then $x, x_{1} \in T$ implies $x \sigma x_{1}$. Thus, if $[x]=\left[x_{1}\right]$ then $x \quad \sigma x_{1}$. Similarly $y \sigma y_{1}$. Therefore $\left[x_{1}\right] \sigma^{\prime}\left[y_{1}\right]$ and $\sigma^{\prime}$ is a well-defined equivalence relation on $S / T$.

It will be shown that $\sigma^{\prime} \in \Sigma_{S / T}$. If $e$ is a left identity modulo $\sigma$ then [e] is a left identity modulo $\sigma^{\prime}$. Furthermore, if $z \in F(S / \sigma)$ then $[e] b^{\prime}[z]$, which implies that $\sigma^{\prime}$ is not the universal relation. Suppose that $\tau^{\prime}$ is a right congruence on $S / T$ which contains $\sigma^{\prime}$. If $x, y \in S$ let $x \tau y$ if and only if $[x] \tau^{\prime}[y]$. Thus $\tau$ is a right congruence on $S$ which contains $\sigma$. Since $\sigma$ is maximal, either $\tau=\sigma$ or $\tau$ is the universal relation on $S$. Thus either $\tau^{\prime}=$ $\sigma^{\prime}$ or $\tau^{\prime}$ is the universal relation on $S / T$. Therefore $\sigma^{\prime} \in \Sigma_{S / T}$. Furthermore, $[a] b^{\prime}[b]$.

Since $\overline{\operatorname{rad}}_{L} S / T=\overline{\operatorname{rad}}_{R} S / T$ then, by the dual of Lemma 5.3, let $\beta^{\prime}$ be a modular maximal left congruence on $S / T$ such that $[a] \beta^{\prime}[b]$. If $x, y \in S$ let $x \beta y$ if and only if $[x] \beta^{\prime}[y]$. Thus $\beta$ is a modular maximal left congruence on $S$ and $T / \beta \subseteq F(S / \beta)$. Since $a \beta b$ then, by the dual of Lemma 5.3, $a \overline{r a d}_{L} S b$. 
Now suppose that $\sigma \in B$. By Lemma 5.5, let $e \in T$ such that $e$ is a left identity modulo $\sigma$. Thus $e a, e b \in T$ and $e a f \mid T e b$. As was seen in the proof of Theorem 5.6, $\sigma \mid T \in \Sigma_{T}$. Since $\overline{\operatorname{rad}}_{L} T=\overline{\operatorname{rad}}_{R} T$ and since $e a \overline{\operatorname{rad}}_{R} T$ $e b$, let $\tau$ be a modular maximal left congruence on $T$ such that $e a f e b$. Let $f$ be a right identity modulo $\tau$. If $x, y \in S$ let $x \gamma_{\tau} y$ if and only if $p x \tau p y$ for each $p \in T$. By work similar to that in the proof of Theorem 5.6, one can show that $\gamma_{\tau}$ is a modular maximal left congruence on $S$ with right identity $f$. Since $e a+e b$ and $e \in T$ then $a f_{\tau} b$. Thus by the dual of Lemma 5.3, $a$ rad $_{L} S b$.

Conversely, one can show that $a \overline{\mathrm{r}}_{\bar{\alpha}} S b$ implies $a \overline{\mathrm{r}}_{R} S b$.

TheOREM 5.8. Let $M^{\circ}(G, I, \Lambda, P)$ be as before. Then $N\left(M^{\circ}\right)=\{0\}$ and if $b, c \in G$ then $(b)_{i \lambda} \overline{\operatorname{rad}}_{R} M^{\circ}(c)_{j \mu}$ if and only if $i, j$ are column-compatible and $\lambda, \mu$ are row-compatible.

Proof. Divide $\Lambda$ into its disjoint classes of row-compatible elements, $\{\Lambda q: q \in Q, 0 \notin Q\}$. Thus $\lambda, \mu \in \Lambda q$ for some $q \in Q$ if and only if $\lambda, \mu$ are row-compatible. If $\omega \in \Lambda$ let $\sigma_{\omega}$ be the right congruence relation on $M^{\circ}$ consisting of the equivalence classes

$$
\begin{aligned}
& x_{0}=\left\{(a)_{i \lambda}: a \in G, p_{\omega i}=0\right\} \cup\{0\}, \\
& x_{q}=\left\{(a)_{i \lambda}: a \in G, p_{\omega i} \neq 0, \lambda \in \Lambda q\right\} \quad \text { for each } q \in Q .
\end{aligned}
$$

It will be shown that

$$
\Sigma_{\mu^{\circ}}=\left\{\sigma_{\omega}: \omega \in \Lambda\right\} .
$$

It is clear that $\sigma_{\omega}$ is an equivalence relation. To show that $\sigma_{\omega}$ is a right congruence on $M^{\circ}$, let $(a)_{i \lambda},(b)_{j \pi}$, and $(c)_{k \mu} \in M^{\circ}$ such that $(a)_{i \lambda} \sigma_{\omega}(b)_{j \pi}$. Since $x_{0}$ is a right ideal of $M^{\circ},(a)_{i \lambda},(b)_{j \pi} \in x_{0}$ implies $(a)_{i \lambda}(c)_{k \mu},(b)_{j \pi}(c)_{k \mu}$ $\in x_{0}$. Suppose that $(a)_{i \lambda},(b)_{j \pi} \in x_{q}$ for some $q \in Q$. Thus $\lambda$ and $\pi$ are row-compatible, which implies that $a p_{\lambda k} c \neq 0$ if and only if $b p_{\pi k} c \neq 0$. Let $v \in Q$ such that $\mu \in \Lambda_{v}$. If $a p_{\lambda k} c \neq 0$ then

$$
(a)_{i \lambda}(c)_{k \mu}=\left(a p_{\lambda k} c\right)_{i \mu} \in x_{v} \text { and }(b)_{j \pi}(c)_{k \mu}=\left(b p_{\pi k} c\right)_{j \mu} \in x_{v} \text {. }
$$

If $a p_{\lambda k} c=0$ then $(a)_{i \lambda}(c)_{k \mu} \in x_{0}$ and $(b)_{j \pi}(c)_{k \mu} \in x_{0}$. In either case, $(a)_{i \lambda}(c)_{k \mu} \sigma_{\omega}(b)_{j \pi}(c)_{k \mu}$, which implies that $\sigma_{\omega}$ is a right congruence on $M^{\circ}$. It is clear that $x_{0}$ is the zero element of $M^{\circ} / \sigma_{\omega}$. Since $P$ is regular, let $m \in I$ such that $p_{\omega m} \neq 0$. Thus $\sigma_{\omega}$ is not the universal relation. Furthermore, it will now be shown that $\left(p_{\omega m}^{-1}\right)_{m \omega}$ is a left identity modulo $\sigma_{\omega}$. Let $(b)_{j \pi} \in M^{\circ}$. If $p_{\omega j} \neq 0$ then 


$$
\left(p_{\omega m}^{-1}\right)_{m \omega}(b)_{j \pi}=\left(p_{\omega m}^{-1} p_{\omega j} b\right)_{m \pi} \sigma_{\omega}(b)_{j \pi} .
$$

If $p_{\omega j}=0$ then $(b)_{j \pi} \in x_{0}$ and $\left(p_{\omega m}^{-1}\right)_{m \omega}(b)_{j \pi}=0 \in x_{0}$, which implies $\left(p_{\omega m}^{-1}\right)_{m \omega}(b)_{j \pi} \sigma_{\omega}(b)_{j \pi}$. Thus $\left(p_{\omega m}^{-1}\right)_{m \omega}$ is a left identity modulo $\sigma_{\omega}$.

By Theorem 1 of [10], let $\gamma$ be a maximal right congruence on $M^{\circ}$ which contains $\sigma_{\omega}$. By Lemmas 4.4 and 4.5, if $(a)_{i \lambda} \in x_{0}$ and $(b)_{j \pi} \in x_{q}$ for some $q \in Q$ then $(a)_{i \lambda} \downarrow(b)_{j \pi}$. By Lemma 4.5, if $(a)_{i \lambda} \in x_{q}$ and $(b)_{j \pi} \in x_{v}$ for $q, v \in Q$ then $(a)_{i \lambda} \gamma(b)_{j \pi}$ implies that $\lambda$ and $\pi$ are row-compatible. But this implies $\Lambda_{q}=\Lambda_{v}$ and $x_{q}=x_{v}$. Thus $\sigma_{\omega}=\gamma$ and $\sigma_{\omega} \in \Sigma_{M^{\circ}}$.

Conversely, let $\tau \in \Sigma_{M^{\circ}}$. Let $(a)_{m \omega}$ be a left identity modulo $\tau$. By Lemmas 4.4 and 4.5, $\tau \subseteq \sigma_{\omega}$. Since $\tau$ is maximal, $\tau=\sigma_{\omega}$. Thus

$$
\Sigma_{M^{\circ}}=\left\{\sigma_{\omega}: \omega \in \Lambda\right\} \text { and } \overline{\operatorname{rad}}_{R} M^{\circ}=\bigcap_{\omega \in \Lambda} \sigma_{\omega} .
$$

Since $P$ is regular, for each $i \in I$ there exists $\omega \in \Lambda$ such that $p_{\omega i} \neq$ 0 . Thus row $i$ is a $\sigma_{\omega}$-nonzero row. Therefore, by Lemma 5.4, $N\left(M^{\circ}\right)=\{0\}$. Furthermore $(a)_{i \mu} \bigcap_{\omega \in \Lambda} \sigma_{\omega}(b)_{i \lambda}$ for each $a, b \in G, i \in I$ if and only if $\lambda$ and $\mu$ are row-compatible. $(a)_{i \mu} \bigcap_{\omega \in \Lambda} \sigma_{\omega}(c)_{j \mu}$ for each $a, c \in G, \mu \in \Lambda$ if and only if $i$ and $j$ are column-compatible. Thus $(b)_{i \lambda} \overline{\operatorname{rad}}_{R} M^{\circ}(c)_{j \mu}$ if and only if $i, j$ are column-compatible and $\lambda, \mu$ are row-compatible.

Since similar theorems may be proved for the left Jacobson radical congruence, we have

Corollary 5.9. $\overline{\operatorname{rad}}_{L} M^{\circ}=\overline{\operatorname{rad}}_{R} M^{\circ}$.

In the previous section, $\operatorname{rad}_{L} M^{\circ}=\operatorname{rad}_{R} M^{\circ}$ if and only if both congruences are the identity relation. However, since $\overline{\operatorname{rad}}_{L} M^{\circ}=\overline{\operatorname{rad}}_{R} M^{\circ}$, then it is not necessarily true that the right Jacobson radical congruence of $M^{\circ}$ equals the right nil radical congruence of $M^{\circ}$.

COROLlARY 5.10. Let $S$ be a semigroup with zero element which satisfies the ascending and descending chain conditions on left and right ideals. Then $\overline{\operatorname{rad}}_{L} S=\overline{\operatorname{rad}}_{R} S$.

Proof. If $N(S)=S$ then $\overline{\operatorname{rad}}_{R} S=\overline{\operatorname{rad}}_{L} S=$ the universal relation on $S$. Assume that $N(S) \neq S$. Let

$$
N(S)=T_{0} \varsubsetneqq T_{1} \varsubsetneqq T_{2} \subsetneq \cdots \subsetneq T_{n}=S
$$

where, for each $i=1,2, \cdots, n, T_{i}$ is an ideal of $S$ such that there is no ideal of $S$ strictly between $T_{i}$ and $T_{i-1}$. Thus each $T_{i} / T_{i-1}$ is either null or 0-simple. If $T_{i} / T_{i-1}$ is null then $\overline{\operatorname{rad}}_{R} T_{i} / T_{i-1}=\overline{\operatorname{rad}}_{L} T_{i} / T_{i-1}=$ the universal relation on $T_{i} / T_{i-1}$. If $T_{i} / T_{i-1}$ is 0 -simple then, by Corollary 2.48 
and Theorem 3.5 of [2], $T_{i} / T_{i-1}$ is isomorphic to a regular Rees matrix semigroup over a group with zero. Therefore, by Corollary 5.9, $\overline{\operatorname{rad}}_{R} T_{i} / T_{i-1}=$ $\overline{\operatorname{rad}}_{L} T_{i} / T_{i-1}$ for each $i=1,2, \cdots, n$.

Since $N(N(S))=N(S)$, then $\overline{\operatorname{rad}}_{R} N(S)=\overline{\operatorname{rad}}_{L} N(S)=$ the universal relation on $N(S)$. Therefore, by Theorem 5.7, $\overline{\operatorname{rad}}_{R} S=\overline{\operatorname{rad}}_{L} S$.

\section{REFERENCES}

1. B. D. Arendt, Semisimple bands, Trans. Amer. Math. Soc. 143 (1969), 133-143. MR 40 \#255.

2. A. H. Clifford and G. B. Preston, The algebraic theory of semigroups. I, Math. Surveys, No. 7, Amer. Math. Soc., Providence, R. I., 1961. MR 24 \#A2627.

3. N. J. Divinsky, Rings and radicals, Math. Exposition, No. 14, Univ. of Toronto Press, Toronto, Ont., 1965. MR 33 \#5654.

4. H.-J. Hoehnke, Structure of semigroups, Canad. J. Math. 18 (1966), 449-491. MR 33 \#5762.

5. - Über das untere und obere Radikal einer Halbgruppe, Math. Z. 89 (1965), 300-311. MR 31 \#3526.

6. Nathan Jacobson, Structure of rings, Amer. Math. Soc. Colloq. Publ., vol. 37, Amer. Math. Soc., Providence, R. I., 1956. MR 18, 373.

7. D. R. LaTorre, Modular congruences and the Brown-McCoy radical for semigroups, Proc. Amer. Math. Soc. 29 (1971), 427-433. MR 43 \#6350.

8. R. H. Oehmke, On maximal congruences and finite semisimple semigroups, Trans. Amer. Math. Soc. 125 (1966), 223-237. MR 34 \#2739.

9. H. Seidel, Über das Radikal einer Halbgruppe, Math. Nachr. 29 (1965), 255-263. MR 32 \#1276.

10. R. E. Slover, Representations of a semigroup, Trans. Amer. Math. Soc. 120 (1965), 417-427. MR 32 \#5765.

11. E. J. Tully, Jr., Representation of a semigroup by transformations acting transitively on a set, Amer. J. Math. 83 (1961), 533-541. MR 25 \#135.

DEPARTMENT OF MATHEMATICS, VIRGINIA POLYTECHNIC INSTITUTE AND STATE UNIVERSITY, BLACKSBURG, VIRGINIA 25061 